\title{
COTIDIANO DE AGENTES COMUNITÁRIOS DE SAÚDE COM IDOSOS SEGUNDO O REFERENCIAL DE CERTEAU
}

\author{
Doane Martins da Silva ${ }^{1}$, Marina Aparecida Chrispim Silva ${ }^{2}$, Dianna Silva de Oliveira ${ }^{2}$, Marilia Alves $^{3}$
}

\begin{abstract}
RESUMO: Objetivou compreender práticas cotidianas de Agentes Comunitários de Saúde na atenção à saúde de idosos. Estudo de caso de abordagem qualitativa, fundamentado no referencial teórico de Certeau acerca do cotidiano, cujos participantes foram oito Agentes de equipes de saúde da família do município de Montes Claros, Minas Gerais, Brasil. Adotou-se a entrevista individual utilizando roteiro semiestruturado. As entrevistas foram realizadas em maio de 2016, gravadas e submetidas à análise de conteúdo. As práticas cotidianas dos Agentes na atenção à saúde de idosos envolvem estratégias e táticas. As visitas domiciliares e os grupos de educação em saúde destacaram-se no cuidado aos idosos. Na prática cotidiana, os Agentes revelaram dificuldades associadas à não efetivação das estratégias do sistema de saúde. Com base nestes achados, torna-se necessária a implementação das estratégias de abrangência nacional para a atenção aos idosos, como subsídio para as práticas dos Agentes, potencializando a atenção à saúde aos usuários.
\end{abstract}

DESCRITORES: Atenção primária à saúde; Agentes comunitários de saúde; Saúde do idoso; Envelhecimento.

\section{DAILY WORK OF COMMUNITY HEALTH WORKERS INVOLVING THE ELDERLY ACCORDING TO CERTEAU'S FRAMEWORK}

ABSTRACT: We aimed to understand daily practices of community health workers in elderly health care. Qualitative case study, based on Certeau's theoretical framework of the daily. The participants were eight workers from family health teams in Montes Claros, Minas Gerais, Brazil. Individual interviews were undertaken using a semistructured script. The interviews were held in May 2016, recorded and submitted to content analysis. The workers' daily practices in elderly health care involve strategies and tactics. The home visits and health education groups stood out in elderly care. In daily practice, the workers revealed difficulties associated with the fact that health system strategies are not put into effect. Based on these findings, nationwide strategies need to be implemented for elderly care to support the workers' practices, enhancing health care for the users.

DESCRIPTORS: Primary health care; Community health workers; Health of the elderly; Aging.

\section{COTIDIANO DE AGENTES COMUNITARIOS DE SALUD CON ANCIANOS SEGÚN EL REFERENCIAL DE CERTEAU}

RESUMEN: La finalidad fue comprender prácticas cotidianas de Agentes Comunitarios de Salude n la atención a la salud del anciano. Estudio de caso de aproximación cualitativa, basado en el referencial teórico de Certeau acerca del cotidiano, cuyos participantes fueron ocho agentes de equipos de salud de la familia del municipio de Montes Claros, Minas Gerais, Brasil. Fue adoptada la entrevista individual mediante un guión semiestructurado. Las entrevistas fueron llevadas a cabo en mayo del 2016, grabadas y sometidas al análisis de contenido. Las prácticas cotidianas de los Agentes en la atención a la salud de ancianos involucran estrategias y tácticas. Las visitas domiciliarias y los grupos de educación en salud se destacaron en el cuidado a los ancianos. En la práctica cotidiana, los agentes revelaron dificultades asociadas a la no efectuación de las estrategias del sistema de salud. Con base en estos hallazgos, se hace necesario implementar las estrategias de alcance nacional para la atención a los ancianos, como apoyo a las prácticas de los agentes, potencializando la atención a la salud de los usuarios.

DESCRIPTORES: Atención primaria de salud; Agentes comunitarios de salud; Salud del anciano; Envejecimiento.

${ }^{1}$ Enfermeira. Doutoranda em Enfermagem. Universidade Federal de Minas Gerais. Belo Horizonte, MG, Brasil.

${ }^{2}$ Discente de Enfermagem. Universidade Federal de Minas Gerais. Belo Horizonte, MG, Brasil.

${ }^{3}$ Enfermeira. Doutora em Enfermagem. Docente de Enfermagem e do Programa de Pós-Graduação em Enfermagem da Universidade Federal de Minas Gerais. Belo Horizonte, MG, Brasil. 


\section{- INTRODUÇÃO}

A Estratégia Saúde da Família (ESF), principal modelo de reorganização da Atenção Primária em Saúde (APS) no Brasil, é porta de entrada preferencial do sistema local de saúde ${ }^{(1)}$. A ESF tem como objetivo produzir nova dinâmica na APS, com definição de responsabilidades entre os serviços de saúde e a população. A equipe básica de saúde da família é composta por médico, enfermeiro, auxiliar de enfermagem e agentes comunitários de saúde (ACS) ${ }^{(1)}$.

Na ESF, a atenção à saúde dos idosos é importante componente do cuidado das equipes de saúde da família e a atuação dos profissionais torna-se essencial pelo seu potencial de intervenção nas necessidades de saúde desta população, que apresenta crescimento acelerado no país, com as mudanças dos padrões demográfico e epidemiológico ${ }^{(2)}$. Este crescimento constitui desafio para as políticas públicas e serviços de saúde ${ }^{(3)}$.

No Brasil, dentre as políticas públicas em saúde direcionadas para a população idosa, destaca-se a mais recente, a Política Nacional de Saúde da Pessoa Idosa (PNSPI) (2). Propõe que a atenção à saúde dos idosos tenha como porta de entrada a APS/ESF, sendo definidas estratégias para a organização dos serviços e estabelecidas ações a serem desenvolvidas pelos profissionais ${ }^{(2)}$.

Com efeito, a ESF é reconhecida como cenário de ações voltadas para o cuidado aos idosos. A atuação dos profissionais em uma comunidade adscrita e a atenção domiciliar possibilitam o conhecimento da realidade do idoso e sua família ${ }^{(4)}$. Reconhece-se, entre outros aspectos, o papel de destaque dos ACS na equipe de saúde da família, foco deste estudo, uma vez que estabelecem mediação e vínculo entre o idoso e a equipe de saúde da família nas unidades básicas de saúde (UBS).

O termo genérico "Agente Comunitário de Saúde" é usado mundialmente para se referir a uma variedade de trabalhadores comunitários. Apesar de diferentes denominações, peculiaridades contextuais, marcos regulatórios e diversidade de atividades, a relação de elo com a comunidade e com o serviço de saúde é característica essencial dos ACS nos diversos países ${ }^{(5)}$.

Estudos $^{(5-7)}$ revelaram a efetividade dos ACS na melhoria da saúde materna e infantil, ampliação do acesso aos cuidados de saúde, diminuição da carga de doença e custos da tuberculose e malária. No Brasil, a atuação dos ACS caracteriza-se por atividades de prevenção de doenças e promoção da saúde, mediante ações domiciliares ou comunitárias, individuais ou coletivas, desenvolvidas em conformidade com as diretrizes do Sistema Único de Saúde (SUS) ${ }^{(8)}$.

Diante do rápido envelhecimento da população brasileira e da necessidade de planejamento e gestão de serviços aos idosos ${ }^{(3)}$, torna-se relevante estudos sobre práticas cotidianas de profissionais da ESF, considerando as múltiplas significações que as estratégias podem assumir nos serviços de saúde, em virtude do confronto com o cotidiano, que é dinâmico.

Certeau $^{(9)}$ conceitualizou cotidiano como um movimento de articulação de práticas do tipo "estratégias" e "táticas". As "estratégias" correspondem às práticas cotidianas derivadas das regras formais. As "táticas" emergem diante das dificuldades de viabilizar as estratégias. Táticas e estratégias interagem e coexistem no cotidiano ${ }^{(10)}$.

Certeau(9) acrescenta, ainda, os conceitos de "lugar" e "espaço" para a compreensão das estratégias e táticas. "Lugar" faz parte do domínio do estratégico e diz respeito às estruturas físicas onde ocorrem as ações dos profissionais (como UBS) ${ }^{(11)}$. O "espaço" (parte de ações táticas) é a prática do lugar ou as formas como os sujeitos o transformam a partir de suas apropriações (como praças públicas) ${ }^{(11)}$. O espaço é um lugar praticado(9).

Assim, parte-se do pressuposto de que, no cotidiano de trabalho com idosos, os ACS seguem aquilo que é normatizado para determinado lugar (as estratégias), mas, diante da confrontação com a realidade cotidiana, instituem novos modos de fazer por meio de táticas, em diferentes espaços, o que expressa a dinamicidade do cotidiano.

O estudo das práticas cotidianas de ACS pode oferecer subsídio para o melhor atendimento às necessidades de idosos na ESF e colocar em prática estratégias governamentais, ampliando o conhecimento sobre diferentes maneiras de fazer (táticas) com os recursos disponíveis, em diferentes 
espaços. Assim, o objetivo deste estudo foi compreender práticas cotidianas de Agentes Comunitários de Saúde na atenção à saúde de idosos.

\section{MÉTODO}

Estudo de caso de abordagem qualitativa, fundamentado no referencial teórico de $\mathrm{Certeau}^{(9)}$ acerca do cotidiano. O estudo de caso visa "captar as circunstâncias e condições de uma situação cotidiana" (12:55). A abordagem qualitativa possibilita "estudar o significado das vidas das pessoas nas condições em que realmente vivem" (13:7).

Estudos em diversas áreas do conhecimento têm adotado este referencial ${ }^{(10,14-15)}$. No contexto da saúde, tem sido utilizado para estudar práticas cotidianas dos profissionais de Serviço de Atendimento Móvel de Urgência ${ }^{(16)}$; analisar abordagens do cotidiano da enfermagem ${ }^{(17)}$; conhecer táticas de usuários e trabalhadoras de programa de saúde mental ${ }^{(18)}$. Contudo, não se conhecem estudos que focalizem práticas cotidianas de ACS na atenção à saúde de idosos.

Os participantes do estudo foram ACS do município de Montes Claros, norte de Minas Gerais, Brasil. O município possuía, à época da coleta de dados, 134 equipes de saúde da família, distribuídas em 115 UBS $^{(19)}$. Foram selecionadas, de modo intencional, duas UBS (denominadas neste estudo de A e B), por concentrarem maior número de usuários com 60 ou mais anos de idade, possuírem maior número de equipes de saude da familia (4 e 3 equipes, respectivamente) e facilidade de acesso aos profissionais.

Em uma primeira abordagem, foi apresentado o projeto para os ACS, feito o convite para participarem da pesquisa, atendendo aos seguintes critérios de inclusão: ser ACS em equipes de saúde da família da zona urbana e atuar por, no mínimo, seis meses na função, considerando as experiências no cotidiano da atenção à saúde de idosos. Como critérios de exclusão: ausência do trabalho por estarem de férias ou licenciados no período da coleta de dados ou se recusarem a participar do estudo.

A coleta de dados foi encerrada com oito entrevistados, considerado número suficiente capaz de propiciar reincidência e complementaridade das informações ${ }^{(20)}$ diante da homogeneidade do grupo, que apresentava pouca diferenciação e características similares das práticas profissionais.

Os dados foram coletados durante o mês de maio de 2016, por meio de entrevista individual utilizando um roteiro semiestruturado com questões referentes à prática cotidiana dos ACS na atenção à saúde de idosos e fatores que influenciam esta atenção na ESF. Para garantir o anonimato dos participantes, as entrevistas foram numeradas na sequência em que ocorreram, atribuindo-se a letra " $E$ " de entrevista. As entrevistas foram gravadas, ouvidas na totalidade, transcritas e submetidas à análise de conteúdo temática ${ }^{(21)}$.

O projeto foi aprovado pelo Comitê de Ética em Pesquisa da Universidade Federal de Minas Gerais, Parecer: 1.486.033/2016.

\section{RESULTADOS}

Os participantes foram do sexo feminino, com idade entre 28 e 49 anos. Em relação à escolaridade, duas possuíam ensino médio completo, uma curso técnico, duas ensino superior em andamento e três haviam concluído o ensino superior. Todas com tempo de atuação variando entre 1 e 9 anos, e cumprem jornada de trabalho de 40 horas.

Os ACS descrevem que suas práticas cotidianas na atenção à saúde de idosos envolvem estratégias e táticas. No âmbito das estratégias, os participantes referiram práticas em consonância com as atividades definidas a priori pela legislação para o lugar ESF: i) cadastramento de famílias, mantendo os dados sobre a população da área atualizados no sistema de informação; ii) visitas domiciliares; iii) grupos de educação em saúde.

Nós iniciamos na parte da manhã, a gente foca mais na questão de fazer cadastro [...]. (E1) 
[...] eu faço as visitas domiciliares [...] tem um sistema E-SUS que a gente tem que digitalizar os dados. Faz grupos [...] às vezes tem um público-alvo. (E3)

A visita domiciliar destacou-se como principal atividade dos ACS no cuidado aos idosos. Nestas visitas, os ACS fornecem orientações relacionadas ao autocuidado, monitoramento de doenças e uso de medicamentos.

[...] às vezes, tem um idoso na área que é difícil vir na unidade [...] a gente tem que acompanhar na casa dele, se está precisando renovar uma receita a gente pede a médica para renovar. E orienta quem cuida para estar atento aos remédios [...]. (E7)

As atividades realizadas na ESF com a participação de ACS, médico e enfermeiro são grupos de educação em saúde para doenças crônicas (sobretudo, diabetes e hipertensão), sendo os idosos a maioria dos participantes nos grupos.

Na realidade o que nós mais desenvolvemos são grupos voltados para hipertenso e diabético, sendo a maioria de idosos [...]. (E5)

Todo mês a gente tem que ter três grupos, exigência da secretaria [...] eu faço grupo de hiperdia [...] o enfermeiro ou a médica faz a palestra, eu só participo organizando [...] a gente fala sobre hipertensão e diabetes, qual a causa, como previne. (E4)

No entanto, foi descrito que as ações educativas não são somente transmissão de orientações, mas se caracterizam pelo compartilhamento de experiências entre usuários e profissionais.

[...] na verdade é uma conversa entre profissionais e a população, não chega a ser uma palestra, é um grupo de discussão, não é aquela coisa de só um falando [...] a gente introduz o assunto, vai vendo as dúvidas e cada um vai relatando experiências. (E5)

Embora as atividades dos ACS sejam determinadas para responder à demanda dos idosos, estes adotam táticas que permitem lidar com as situações do cotidiano. Os profissionais têm de usar táticas essencialmente para atender às necessidades dos idosos. Estas táticas são:

i) Sair dos lugares formais (UBS) e utilizar espaços no território que fiquem mais acessíveis aos idosos (como igrejas, parques, domicílio dos ACS ou dos idosos) para realizar grupos de educação em saúde;

[...] a gente não tem um local para fazer o grupo [...] a gente pede na casa de famílias para fazer o grupo ou eu faço lá em casa [...]. (E7)

Hoje estou com o grupo Bem-Estar. A gente escolhe um tema no mês e aborda [...] tenta fazer dinâmicas, brincadeiras. O local é a igreja, mais perto para eles [...]. (E8)

A gente orienta os idosos com hipertensão e diabetes para ir à Praça do Parque das Mangueiras, uma praça bem próxima. (E1)

ii) Adequar a realização das visitas domiciliares às necessidades dos idosos. Os ACS confrontamse com idosos com limitações funcionais que vivem sozinhos e se mostram sensíveis a esta situação, valendo-se de uma frequência maior de visitas e de uma maior cautela na realização destas;

Os idosos requerem atenção especial [...] No mês eu faço a busca ativa deles, chego a fazer até quatro visitas porque fico preocupada dele morar sozinho e acontecer alguma coisa. (E2)

iii) Adequar as práticas de educação em saúde, sobretudo usando atividades lúdicas e valendo-se da presença do médico, enfermeiro e da oferta de procedimentos (aferição de pressão arterial e glicemia) como formas convidativas.

A gente vai às casas, avisa que vai ter o grupo e que vai ter a presença do médico ou do enfermeiro [...] que vai ter aferição de pressão arterial e de glicemia [...] nos grupos a gente faz brincadeiras e bingos para poder estar brincando e distraindo. (E3)

Apesar das táticas criadas pelos ACS no cotidiano de trabalho da ESF, foram descritas dificuldades na prática cotidiana com idosos relacionadas com: i) o número de usuários cadastrados por equipe; 
ii) demora em agendar consulta e exames especializados. Essas dificuldades estão associadas à não efetivação das estratégias definidas pelo Ministério da Saúde (nível macro) para a atenção ao idoso ou à demora dessas estratégias em se expressar, a contento, na ESF.

[...] mais profissionais médicos e enfermeiros para atender porque cada médico atende cerca de 3.500 pessoas [...] porque o médico e o enfermeiro, por terem outras demandas e dar conta de toda a área, não têm como irem todo mês às visitas. (E3)

[...] o médico atende aí precisa de um exame especializado, mas demora a dar continuidade ao tratamento porque não tem aquele exame, a cota de exames e para consultas com especialistas é pequena [...].(E5)

Os ACS referiram ainda: iii) a falta de capacitação para atuarem na área de envelhecimento e saúde do idoso. As capacitações são pontuais, voltadas para doenças crônicas e cuidados nos casos de maior vulnerabilidade, como gestantes e crianças, alvos prioritários.

[...] uma coisa muito rápida, mas teve capacitação [...] a gente fez curso de cuidador, a assistente social fez capacitação sobre o estatuto do idoso [...] a enfermeira falou sobre quedas de idosos e melhorias para adequar banheiro, uso de tapete, iluminação. (E6)

Capacitação que eu me lembre não teve. Porque teve os Caminhos do Cuidado que é para a maternidade, mas para os idosos eu acho que não teve. (E3)

\section{DISCUSSÃO}

Este estudo explorou práticas cotidianas de ACS na atenção à saúde de idosos, considerando o referencial teórico de Certeau ${ }^{(9)}$. Os resultados revelam que as práticas dos ACS envolvem estratégias e táticas e foram descritas dificuldades na prática cotidiana com idosos, relacionadas à não efetivação das estratégias definidas para a atenção à saúde da população idosa.

As atribuições dos ACS estão expressas nas estratégias do Ministério da Saúde, passam por estruturas das secretarias municipais de saúde e tem-se na APS/ESF o lugar destinado à execução das ações estratégicas previamente definidas. Assim, o trabalho dos ACS envolve as práticas cotidianas do tipo estratégias, baseadas em atividades definidas a priori, que abrangem: trabalhar com adscrição de famílias em microáreas; acompanhar, por meio de visita domiciliar, famílias e indivíduos sob sua responsabilidade; desenvolver atividades de promoção da saúde, prevenção das doenças e agravos e de vigilância à saúde, por meio de visitas domiciliares e ações educativas individuais e coletivas nos domicílios e na comunidade ${ }^{(1)}$.

A visita domiciliar destacou-se como principal prática dos ACS direcionada aos idosos. Essa visita possibilita conhecer in loco a realidade e as necessidades dos idosos e de sua família, a identificação e busca ativa de idosos frágeis, o que possibilita o planejamento das ações a partir da realidade, de mediação entre idoso-família e as equipes de saúde da família ${ }^{(22)}$. Os idosos frequentemente são afetados por doenças crônicas (comorbidades), dependência e limitações funcionais, demandando, geralmente, algum cuidador ${ }^{(23)}$, o que reforça a vista domiciliar dos ACS como importante ferramenta de cuidado ao binômio idoso-família.

A prática de realização de grupos de educação em saúde foi relatada pelos ACS dentre as ações que incluem os idosos. No cotidiano de equipes de saúde da família são frequentes os grupos de educação em saúde (como hipertensão, diabetes, gestantes, tabagistas) e os profissionais consideram que os idosos estão incluídos nos grupos de hipertensão e diabetes, pois a maior parte dos participantes dos grupos é idosa. O trabalho em grupo com os idosos é um espaço complementar da consulta individual, de troca de informações, de oferecimento de orientação e de educação em saúde ${ }^{(24)}$.

Entretanto, o trabalho em grupo relatado pelos ACS, pautado no repasse de orientações sobre doenças crônicas, reproduz e reforça o modelo biomédico doença-adoecimento. $\mathrm{O}$ enfoque nas doenças crônicas pode estar associado ao fato de os idosos serem considerados como grupos de risco para essas doenças, especialmente a hipertensão e o diabetes mellitus ${ }^{(25)}$.

Por outro lado, o compartilhamento de experiências nos grupos foi revelador como experiência 
de mudança de comportamento. Estudo revela que ações de educação em saúde podem possibilitar acesso à informação e troca de experiências, bem como contribuir para a promoção da saúde e prevenção de doenças ${ }^{(26)}$.

As práticas do tipo táticas no cotidiano de trabalho dos ACS parecem ocorrer quando, diante da confrontação com a realidade cotidiana, as estratégias não são viáveis ou, por vezes, não é possível realizá-las como estabelecidas a priori e, então, os profissionais valem-se de táticas, muitas vezes silenciosas, e se aproveitam das ocasiões favoráveis ${ }^{(9)}$.

Os ACS revelaram diferentes táticas no cotidiano de trabalho com os idosos, como a tática de apropriação de espaços no território diante da necessidade de lidarem com a ausência de um lugar específico nas UBS, para a realização das atividades de grupo. Essa tática, portanto, impõe-se na necessidade do cotidiano ${ }^{(9)}$.

Os ACS se aproveitam de ocasiões favoráveis (as possibilidades permitidas pelas configurações espaciais disponíveis na comunidade) e criam nesses espaços oportunidades para as atividades de grupo, tornando-os, assim, lugares praticados ${ }^{(9)}$, o que revela outras possíveis maneiras de fazer as atividades educativas em grupos, que não necessariamente circunscrevem-se em demarcações definidas de lugares: elas acontecem, graças à tática dos ACS, em diferentes espaços.

Seguem-se as táticas nas maneiras dos ACS adequarem a realização das visitas às necessidades dos idosos. Mesmo em meio à demanda de acompanhar todos os usuários e famílias de sua área de abrangência, os ACS disponibilizam tempo para aumentar a frequência das visitas aos idosos, considerando que há idosos que residem sozinhos, o que é utilizado para definir a prioridade das visitas.

A visita domiciliar é uma das principais atividades preconizadas pelo Ministério da Saúde para os ACS e devem ser programadas em conjunto com a equipe, considerando os critérios de risco e vulnerabilidade, de modo que famílias com maior necessidade sejam visitadas mais vezes, mantendo como referência a média de uma visita/família/mês ${ }^{(1)}$.

As táticas foram reveladas ainda nas maneiras dos ACS adequarem as práticas de educação em saúde aos idosos. Para incluírem os idosos nos grupos, valem-se, taticamente, da presença do médico, enfermeiro e da oferta de procedimentos, como aferição de pressão arterial e glicemia, e atividades lúdicas como formas convidativas. Estudo realizado com usuários frequentadores de um grupo de hipertensos e diabéticos na ESF revelou que atividades lúdicas foram eficazes para promover a aprendizagem de forma descontraída e motivavam a participação nos encontros, sendo mais produtivas em relação à compreensão dos assuntos abordados ${ }^{(27)}$.

Entre as dificuldades na prática cotidiana com idosos, foi descrito o número de usuários cadastrados por equipe de saúde da família, embora a população adscrita estivesse dentro dos limites do Ministério da Saúde ${ }^{(1)}$. Cada equipe de saúde da família deve ser responsável por, no máximo, 4.000 pessoas, sendo 3.000 a média recomendada, considerando o grau de vulnerabilidade das famílias do território(1).

Entretanto, este número foi considerado elevado e um dificultador para a realização de visitas domiciliares regulares aos idosos por parte de médicos e enfermeiros. O excesso de usuários adscritos dificulta as ações de prevenção e promoção de saúde, principalmente para adultos e idosos; a sobrecarga de usuários inviabiliza que as equipes invistam maior tempo em práticas preventivas ${ }^{(28)}$.

A necessidade de redução dos usuários para cerca de 2.500 pessoas, em média, por equipe foi revelada na experiência brasileira e também de outros países ${ }^{(29)}$. Outros fatores, como as baixas condições sociais de muitos brasileiros, a vasta extensão territorial do país e o trabalho em equipe, também apontam para esta redução ${ }^{(29)}$.

A demora na marcação de exames e o acesso a consultas com especialistas é uma preocupação dos ACS, o que revela limitações do sistema de saúde, e não apenas do cenário estudado. A demora na marcação de consultas, a falta de especialistas e dificuldades na regulação do sistema municipal de saúde são fatores que influenciam o acesso aos serviços de saúde ${ }^{(30)}$. Desse modo, os municípios devem organizar a rede assistencial com prerrogativa de acesso a exames e consultas especializadas.

A ausência de capacitação para os ACS na área de envelhecimento e saúde do idoso também foi 
uma das dificuldades apontadas. A insuficiente capacitação de profissionais de equipes de saúde da família para a atenção à saúde do idoso foi revelada em outro estudo(28), o que dificulta a abordagem de questões características desta população.

Na PNSPI (2), tem-se, dentre as diretrizes, a formação e educação permanente dos profissionais de saúde do SUS na área de saúde do idoso, porém, diferentemente do recomendado, os ACS não referiram esta formação. Compreende-se, portanto, a necessidade da efetiva implementação das diretrizes expressas na PNSPI.

O estudo foi realizado em um município, com número reduzido de ACS e, por isso, possui limitações quanto à generalização dos resultados. Desta forma, sugerem-se novas investigações acerca dessa temática, incluindo outros profissionais da equipe de saúde da família, suas práticas e saberes na atenção à saúde de idosos no contexto da ESF.

\section{CONCLUSÃO}

Este estudo revelou que as práticas cotidianas dos ACS na atenção à saúde de idosos envolvem estratégias e táticas e foram descritas dificuldades na prática cotidiana com idosos. As dificuldades foram relacionadas à não implementação das estratégias previstas em nível nacional para a atenção ao idoso. A realização de visitas domiciliares e de grupos de educação em saúde destacaram-se no cuidado aos idosos.

O confronto com a realidade demandou dos ACS a utilização de táticas, como a apropriação de espaços no território para a realização das atividades de grupo. Os ACS se referiram à ausência de capacitações para o atendimento aos idosos e oferta insuficiente de exames e consultas com especialistas para usuários idosos.

\section{- REFERÊNCIAS}

1. Ministério da Saúde (BR). Portaria n. 2.488, de 21 de outubro de 2011. Aprova a Política Nacional de Atenção Básica, estabelecendo a revisão de diretrizes e normas para a organização da Atenção Básica, para a Estratégia Saúde da Família (ESF) e o Programa de Agentes Comunitários de Saúde (PACS). Brasília, Diário Oficial da União, 22 out 2011.

2. Ministério da Saúde (BR). Portaria n. 2.528, de 19 de outubro de 2006. Aprova a Política Nacional de Saúde da Pessoa Idosa (PNSPI). Brasília, Diário Oficial da União, 19 out 2006.

3. Miranda GMD, Mendes ACG, Silva ALA. Population aging in Brazil: current and future social challenges and consequences. Rev. bras. geriatr. gerontol. [Internet] 2016;19(3) [acesso em 02 ago 2017]. Disponível: http://dx.doi. org/10.1590/1809-98232016019.150140.

4. de Oliveira JCA, Tavares DMS. Atenção ao idoso na estratégia de Saúde da Família: atuação do enfermeiro. Rev. esc. enferm. USP. [Internet] 2010;44(3) [acesso em 15 ago 2017]. Disponível: http://dx.doi.org/10.1590/S008062342010000300032 .

5. World Health Organization (WHO). Global Health Workforce Alliance. Global experience of community health workers for delivery of health-related millennium development goals: a systematic review, country case studies and recommendations for scaling up. Geneva: WHO/ Global Health Workforce Alliance; 2010.

6. Giugliani C, Harzheim E, Duncan MS, Duncan BB. Effectiveness of community health workers in Brazil: a systematic review. J Ambul Care Manage. [Internet] 2011;34(4) [acesso em 20 jan 2017]. Disponível: http://dx.doi. org/10.1097/JAC.0b013e31822cbdfd.

7. Giugliani C, Duncan BB, Harzheim E, Lavor ACH, Lavor MC, Machado MM, et al. Community health workers programme in Luanda, Angola: an evaluation of the implementation process. Hum Resour Health. [Internet] 2014;(12) [acesso em 23 jan 2017]. Disponível: https://human-resources-health.biomedcentral.com/ articles/10.1186/1478-4491-12-68.

8. Brasil. Lei n. 11.350, de 5 de outubro de 2006. Regulamenta o §5o do art. 198 da Constituição, dispõe sobre o 
aproveitamento de pessoal amparado pelo parágrafo único do art. $2^{\circ}$ da Emenda Constitucional n. 51, de 14 de fevereiro de 2006, e dá outras providências. Diário Oficial da União, 6 out 2006.

9. Certeau M. A Invenção do cotidiano: 1. Artes de fazer. 20ª ed. Petrópolis (RJ): Vozes; 2012.

10. O'Brien M. The Tactics of Mobile Phone Use in the School-Based Practices of Young People. Anthropol Action. [Internet] 2009;(16) [acesso em 03 ago 2017].Disponível: http://dx.doi.org/10.3167/aia.2009.160104.

11. Schneider LC. Lugar e não-lugar: espaços da complexidade. Ágora. [Internet] 2015;17(1) [acesso em 03 ago 2017]. Disponível: http://dx.doi.org/10.17058/agora.v17i1.5311.

12. Yin RK. Estudo de caso: planejamento e métodos. 5ª ed. Porto Alegre: Bookman; 2015.

13. Yin RK. Pesquisa qualitativa do início ao fim. Porto Alegre: Penso; 2016.

14. Thirlway F. Everyday tactics in local moral worlds: E-cigarette practices in a working-class area of the UK. Soc Sci Med. [Internet] 2016;(170) [acesso em 03 ago 2017]. Disponível: http://dx.doi.org/10.1016/j.socscimed.2016.10.012.

15. Benavides ACP. Estelas de trayectorias esparcidas: las tácticas indígenas em el contexto de las misiones. Colombia, 1880-1930. Mem Soc. [Internet] 2016;20(41) [acesso em 03 ago 2017]. Disponível: http://dx.doi. org/10.11144/Javeriana.mys20-41.etet.

16. Araújo MT. Práticas cotidianas do serviço de atendimento móvel [tese]. Belo Horizonte (MG): Universidade Federal de Minas Gerais; 2013.

17. Santos DS, Ribeiro HCTC, Araujo MT, Alves M, Carrieri AP. What is really important to think about everyday life in nursing?: an integrative review. Rev. enferm. UFPE on line. [Internet] 2014;8(10) [acesso em 02 ago 2017]. Disponível: http://www.revista.ufpe.br/revistaenfermagem/index.php/revista/article/view/6008.

18. Schmidt MLS. Produções táticas de usuários e trabalhadoras de programa de saúde mental: estudo etnográfico num centro de saúde escola da zona oeste de São Paulo. Saúde Soc. [Internet] 2013;22(4) [acesso em 02 ago 2017]. Disponível: http://dx.doi.org/10.1590/S0104-12902013000400011.

19. Cadastro Nacional de Estabelecimentos de Saúde (CNES). Estabelecimentos de Saúde do Município: Montes Claros. [Internet] 2016 [acesso em 14 ago 2016]. Disponível: http://cnes2.datasus.gov.br/Mod_Ind_Unidade.asp? VEstado=31\&VMun=314330\&VComp=201607.

20. Minayo MCS. Amostragem e saturação em pesquisa qualitativa: consensos e controvérsias. Rev. Pesqui. Qual. [Internet] 2017;5(7) [acesso em 15 ago 2017]. Disponível: http://rpq.revista.sepq.org.br/index.php/rpq/article/ view/82.

21. Bardin L. Análise de conteúdo. Lisboa: Edições 70; 2011.

22. Magalhães KA, Giacomin KC, dos Santos WJ, Firmo JOA. A visita domiciliária do agente comunitário de saúde a famílias com idosos frágeis. Ciênc. saúde coletiva. [Internet] 2015;20(12) [acesso em 01 ago 2017]. Disponível: http://dx.doi.org/10.1590/1413-812320152012.07622014.

23. Tomomitsu MRSV, Perracini MR, Neri AL. Fatores associados à satisfação com a vida em idosos cuidadores e não-cuidadores. Ciênc. saúde coletiva. [Internet] 2014;19(8) [acesso em 01 ago 2017]. Disponível: http://dx.doi. org/10.1590/1413-81232014198.13952013.

24. Ministério da Saúde (BR). Departamento de Atenção Básica. Secretaria de Atenção à Saúde. Envelhecimento e saúde da pessoa idosa - caderno de atenção básica n. 19. Brasília: Ministério da Saúde; 2006.

25. Gerhardt PC, Borghi AC, Fernandes CAM, Mathias TAF, Carreira L. Tendência das internações por Diabetes Mellitus e Hipertensão Arterial Sistêmica em idosos. Cogitare Enferm. [Internet] 2016;21(4) [acesso em 04 ago 2017]. Disponível: http://dx.doi.org/10.5380/ce.v21i4.44912.

26. Pereira AV, Vieira ALS, Amâncio Filho A. Grupos de educação em saúde: aprendizagem permanente com pessoas soropositivas para o HIV. Trab. educ. Saúde (Online). [Internet] 2011;9(1) [acesso em 19 jan 2017]. Disponível: http://dx.doi.org/10.1590/S1981-77462011000100003. 
de educação em saúde para o saber de pessoas com hipertensão. Rev. bras. enferm. [Internet] 2014;67(3) [acesso em 31 jul 2017]. Disponível: http://dx.doi.org/10.5935/0034-7167.20140045.

28. da Motta LB, de Aguiar AD, Caldas CP. Estratégia de saúde da família e Atenção ao idoso: experiência em três municípios brasileiros. Cad. Saúde Pública. [Internet] 2011;27(4) [acesso em 31 jul 2017]. Disponível: http://dx.doi. org/10.1590/S0102-311X2011000400017.

29. Tesser CD, Norman AH. Repensando o acesso ao cuidado na Estratégia Saúde da Família. Saúde Soc. [Internet] 2014;23(3) [acesso em 01 ago 2017]. Disponível: http://dx.doi.org/10.1590/S0104-12902014000300011.

30. Viegas APB, Carmo RF, da Luz ZMP. Fatores que influenciam o acesso aos serviços de saúde na visão de profissionais e usuários de uma unidade básica de referência. Saúde Soc. [Internet] 2015;24(1) [acesso em 04 ago 2017]. Disponível: http://dx.doi.org/10.1590/S0104-12902015000100008. 